\title{
CONTRAST ENHANCED ULTRASOUND IN CORRELATION WITH HIGH RESOLUTION ADVANCED ULTRASOUND IN THYROID INCIDENTALOMA
}

\author{
Ameen M. D1, Naveen $S^{2}$, Kumaran $L^{3}$
}

${ }^{1}$ Associate Professor, Department of Radiodiagnosis.

${ }^{2}$ Associate Professor, Department of Radiodiagnosis.

${ }^{3}$ Assistant Professor, Department of Radiodiagnosis.

\begin{tabular}{l} 
ABSTRACT \\
\hline BACKGROUND \\
Solitary thyroid nodule in contrary to multiple lesions is uncommon. If sonologically detectable, irrespective of sex, age group \\
above 45 years and in non-endemic areas the malignancy rates are high. CEUS is widely done in other Asian countries, seldom used \\
in India. As cost-effective contrast study unlike CT and MRI contrast with least adverse reactions will really be a value added tool in \\
future and adjunct to routine real-time 2D ultrasonography. \\
$\quad$ The objective of the study is to study the CEUS in correlation with high resolution advanced ultrasonography in diagnosis of \\
solitary thyroid nodule.
\end{tabular}

\section{MATERIALS AND METHODS}

We have decided the sample size of 33 based on previous one year record in our hospital. We enrolled symptomatic patients referred to Radiology Department for ultrasonography from other different specialities, of which 18 cases were with solitary lesions and another 15 cases were with multiple lesions. Contrast agent Sonovue was available in powdered form, mixed with 40 $60 \mathrm{~mL}$ saline for maximum contrast and given according to body weight of the participants. To find the significance in categorical data, Chi-square test was used. The p-value $<0.05$ is considered as significant level.

\section{RESULTS}

There were 17 females and 16 males with overall mean (+SD) age being $43.55(+5)$ years. Mean age for female and male was 43.5 years and 54.3 years respectively. Mean (+SD) size of nodule was $2.5(+1.5) \mathrm{cm}$ and commonly seen on the right side as compared to the left side.

\section{CONCLUSION}

CEUS prove to be definitely adjunct to routine real-time ultrasonography in challenging and problem solving solitary thyroid nodule. Future prospects are yet to be defined for CEUS to include as value added tool along with USG in routine ultrasonography screening.

\section{KEYWORDS}

Contrast Enhanced Ultrasonography (CEUS); Ultrasonography (USG); Solitary Thyroid Nodule; Future Prospects.

HOW TO CITE THIS ARTICLE: Ameen MD, Naveen S, Kumaran L. Contrast enhanced ultrasound in correlation with high resolution advanced ultrasound in thyroid incidentaloma. J. Evolution Med. Dent. Sci. 2018;7(01):5-8, DOI: 10.14260/jemds/2018/2

\section{BACKGROUND}

Thyroid nodules are commonly benign and the reported prevalence widely varies depending on the population studied and the methods used to detect the nodules.(1) Globally, thyroid cancer is increasing rapidly and resulted in 36,000 fatalities in 2010, an increase from 24,000 in 1990, although 5-year survival rates are high following treatment. Cancer incidence represents a substantial health burden. $(2,3)$ Ultrasound (US) is an accepted standard diagnostic method for the detection of thyroid nodules worldwide.(4) However, previous studies found extreme variations in the assessment of thyroid nodules by US.(5) Conventional US has a moderate accuracy for characterising the nature of thyroid nodules, thus supplementary diagnostic methods including radionuclide scanning and fine-needle aspiration (FNA)

'Financial or Other Competing Interest': None.

Submission 05-12-2017, Peer Review 16-12-2017,

Acceptance 19-12-2017, Published 01-01-2018.

Corresponding Author:

Dr. Ameen M. D,

Flat 1C, No. 9, Swaraj Manor Leithe Castle

Centre Street, Santhome, Chennai-600028.

E-mail:drameenmdrd@rocketmail.com

DOI: $10.14260 /$ jemds $/ 2018 / 2$ biopsy are employed for improved clinical evaluation of thyroid nodules.(4) With the introduction of contrast enhanced ultrasound (CEUS) and real-time elastography (RTE), promising results have been reported for better accuracy in differentiating between benign and malignant thyroid nodules. Thyroid nodules are commonly encountered lesions and have been observed in $50 \%$ of autopsied patients.(1) The estimated annual incidence rate of $0.1 \%$ in the ultrasonography (US) suggests 300,000 newly diagnosed nodules as of 2016. CEUS involves the use of a contrast medium that enhances the diagnostic imaging capabilities of traditional medical sonography and is a milestone for diagnostics in liver tumours with recent studies evaluating similar application of CEUS in characterising thyroid gland tumours. CEUS is also amenable to combination approaches and dynamical evaluation of microcirculation with CEUS in combination with colour-coded and power Doppler sonography was demonstrated to yield reliable preoperative results in the characterisation of thyroid adenomas. $(6,7,8)$ Tumours are the typical lesions which depend on blood vessels. Recently, colour Doppler and power Doppler have been the most commonly used methods to detect blood vessels of tumours. Their larger blood vessels can be displayed by Doppler ultrasound, but the microvessels with low speed and flow cannot. Sonovue is a stabilised 
microbubble preparation containing sulphur hexafluoride with an average diameter of $2.5 \mu \mathrm{m}$. Microbubble can flow in the microcirculation and resonate at a low mechanical index. The microvascular perfusion of the tumour can be displayed clearly by using low energy acoustic emission and pulse inversion harmonic imaging. CEUS has made a major improvement in the diagnosis and differential diagnosis of focal liver lesions. Previously, Bartolotta et al studied 18 cases of solitary thyroid nodule by CEUS and the results showed that the diagnosis of thyroid nodules was feasible.(9) Solitary thyroid nodule in contrary to multiple lesions are uncommon. Multinodular goitres and Colloid goitres are most frequently seen as sonological abnormality in endemic regions of India. As Solitary thyroid nodules are being more often, further diagnostic approach seems to be FNAC modality of choice. As FNAC seems to be invasive, poor sampling results in negative study. Moreover, thyroid FNAC related complications are also encountered. In future to prefer non-invasive modality, CEUS can be a value added tool in diagnosing benign versus malignant thyroid nodule. If sonologically detectable, irrespective of sex ratio, age group above 45 years and in non-endemic areas, malignancy rates are high. CEUS is widely done in other Asian countries, seldom used in India. As cost-effective contrast study, unlike CT and MRI contrast with least adverse reactions will really be a value added tool in future and adjunct to routine realtime 2D Ultrasonography. The purpose of the study is to prove the efficacy of CEUS as value added tool in solitary thyroid nodule and to avoid invasive modality choice in future. Our study focused on exploring the observed indicators of contrast enhancement patterns of solitary thyroid nodules and designed to evaluate diagnostic value between malignant and benign thyroid nodules.

\section{MATERIALS AND METHODS}

There were about 30 cases of incidentaloma in the record in the previous year at our hospital's radiology department. In the current year, we expect to get about 33 cases $(10 \%$ increases) in the same time period. Accordingly, 33 cases were enrolled in the study during data collection period from January 2017 to October 2017. We used interview schedule, which contains two sections. The section one contains sociodemographic variables and section two contains variables for recording the reports of imaging including USG and CEUS. All symptomatic patients of thyroid nodules were referred to Radiology department for ultrasonography, of which 18 cases were solitary lesions and 15 cases with multiple lesions were included in the study. After obtaining written consent, 18 patients were studied by CEUS and 15 patients were studied by high resolution ultrasonography for comparison.

\section{Image Protocol}

All patients were examined using a system equipped with Siemens Sequoia 512 colour Doppler ultrasound system unit (probe model 15 L8WS with the band width of 8fdt MHz), which was used as taking in a contrast pulse sequencing (CPS). Initially, patients were assessed on a routine US examination in supine position with the neck fully exposed. Thyroid nodules were evaluated for location, size, margin, internal echo, evenness and calcification. Contrast pulse sequencing was also applied with probe emission frequency of $9-12 \mathrm{MHz}$, mechanical index of 0.08 , imaging depth of 50 $\mathrm{mm}$ and $50 \%$ image gain. Contrast agent Sonovue available in powdered form was mixed with $40-60 \mathrm{~mL}$ saline for maximum contrast and given according to body weight of the patients. Sonovue was injected as an intravenous bolus of 2.4 $\mathrm{mL}$ per subject via an antecubital vein followed by additional $5 \mathrm{~mL}$ of $0.9 \%$ sodium chloride. Subsequent to contrast agent injection, harmonic gray scale CEUS was applied to scan the thyroid gland and the nodule for at least 150 secs and the dynamic images were recorded. The patients' necks were hyperextended to expose the thyroid area. Scanning was performed by one experienced sonographer, who was asked to evaluate the nodule location, size and Doppler flow signals. The standard view of CEUS was the section, which showed the most abundant blood flow signals within the lesion by power Doppler and the whole thyroid nodule and as much of the surrounding thyroid as possible. Focus was located in the trailing edge of the lesion, and the gain was adjusted to display only the boundaries of the lesion. Then, CPS was started and $2.5 \mathrm{~mL}$ of US contrast agent.

\section{Statistical Analysis}

Descriptive statistics like percentage, mean and standard deviation, etc. were used. The inferential statistics like Chisquare test was used for finding out association using categorical data. The collected data were analysed using SPSS Software 23.0 version. The ' $p$ ' value was taken $<0.05$ as level of significance.

\section{RESULTS}

We have collected information relevant to the study topics from the selected individuals and recorded in the interview schedule. There were 17 females and 16 males (Figure 1) presenting with solitary nodule with overall mean (+SD) age of $43.55(+5)$ years. Mean age for female and male was 43.5 years and 54.3 years respectively. Mean $(+\mathrm{SD})$ size of nodule was $2.5(+1.5) \mathrm{cm}$ and is commonly seen on the right side as compared to the left side. (Table 1) There were four categories of benign and malignant nodules detection delineated by CEUS technique. In Category 1: $6.0 \%$ was benign, $9.0 \%$ was malignant. Category 2: Also showing 6.0\% as benign and $3.0 \%$ as malignant. Category 3: $6.0 \%$ was benign and $9.0 \%$ was malignant. Category $4: 9.0 \%$ was benign and $3.0 \%$ was malignant (Fig. 2). But we could not find any significant association of category-wise differentiation in diagnosis of benign and malignant thyroid tumour (Table 1).

\section{Image Analysis and Interpretation}

The differential diagnosis of malignant or benign thyroid nodules was based on the results obtained from CEUS and compared with high resolution US (HRUS). CEUS diagnostic algorithm was grouped into 4 categories, after giving contrast study for contrast study analysis. Category 1: Degree of enhancement (smooth or coarse enhancement). Category 2: Surface enhancement (regular or irregular). Category 3: Wash-in time (in seconds how much time, contrast reach nodule). Category 4: Wash-out time (in seconds how much time, contrast wash out from nodule). Figure 4 showing the CEUS' more morphological delineation in predicting solitary nodule pathology as compared with high resolution ultrasound. According to Bartolotta, enhancement patterns of thyroid nodules with Sonovue and found that enhancement patterns of thyroid nodules were closely related to lesion size. Figure 5 is also showing indeterminate lesion in high resolution ultrasonography, delineated better results in contrast enhanced ultrasonography. 


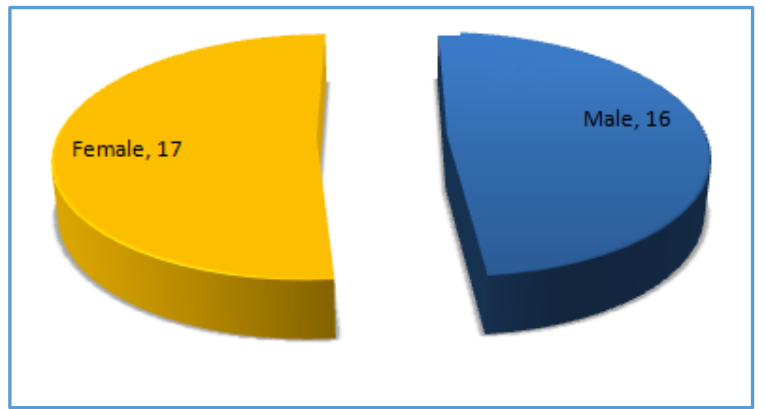

Figure 1. The Distribution of Participants according to Sex

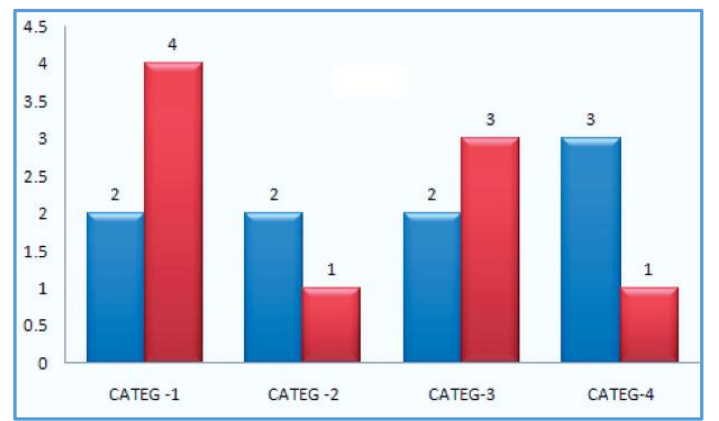

Figure 2. The Distribution of Thyrpoid Tumours

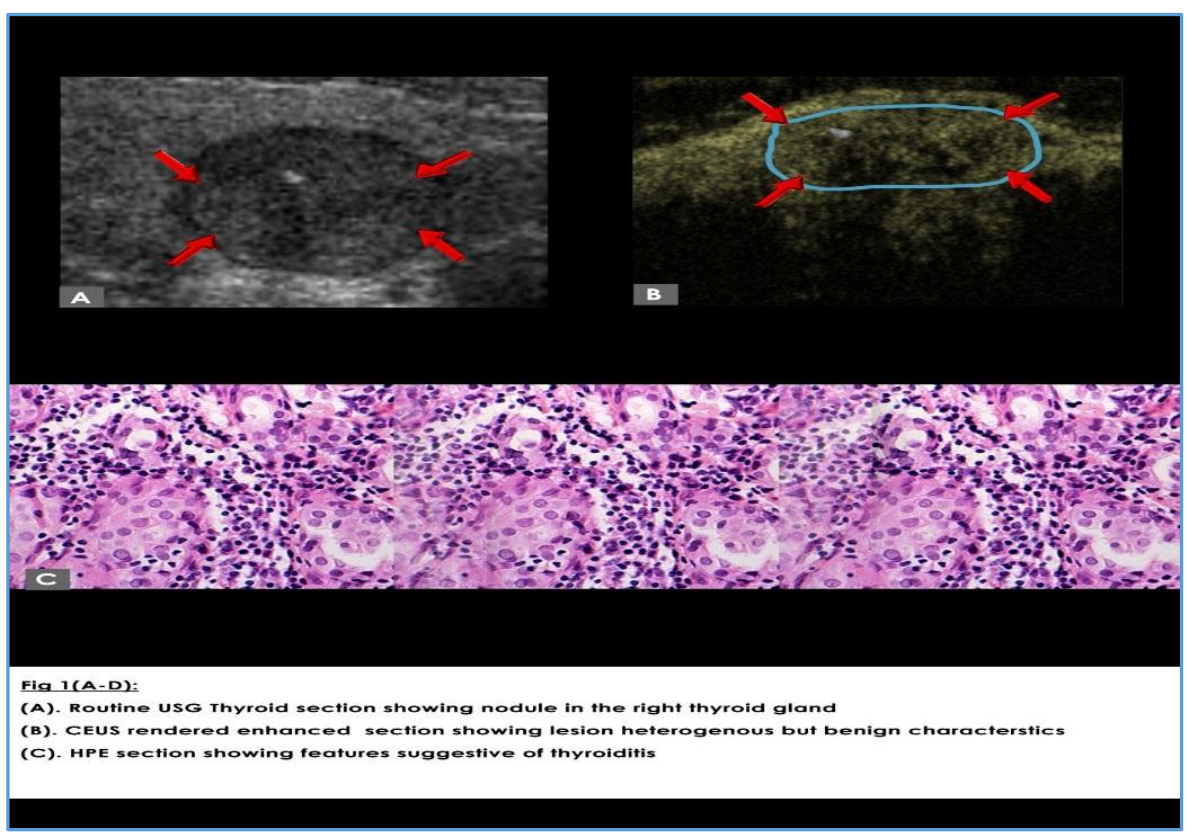

Figure 3. Showing CEUS more Morphological Delineation in Predicting Solitary Nodule Pathology as compared with High Resolution Ultrasound

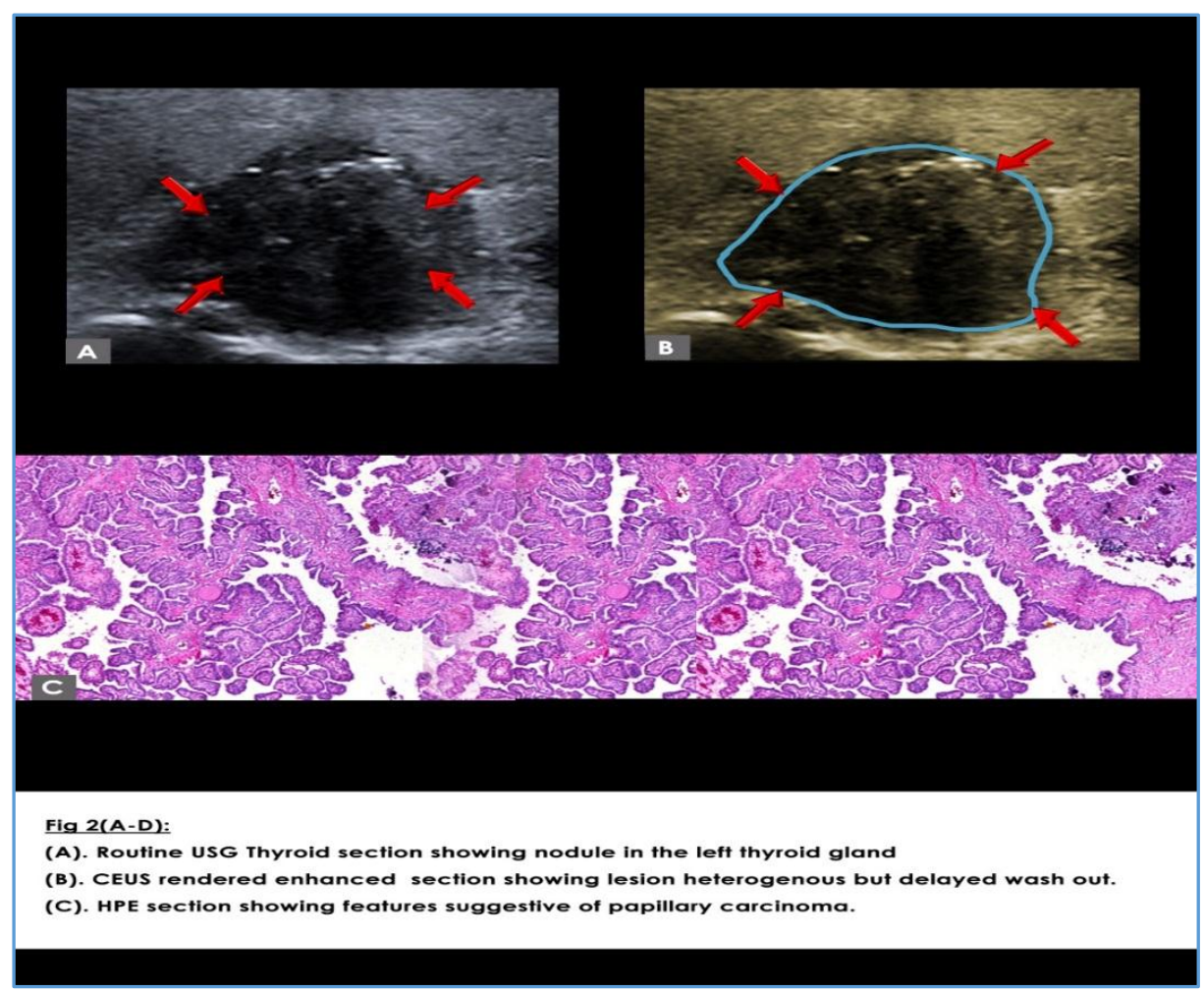

Figure 4. Showing Indeterminate Lesion in High Resolution Ultrasonography, Delineated better Results in Contrast Enhanced Ultrasonography 


\begin{tabular}{|c|c|c|c|}
\hline Variables & Benign, N (\%) & Malignant, N (\%) & \multirow{2}{*}{ P value } \\
\hline Cat-I & $2(22.22)$ & $4(44.44)$ & \\
\cline { 1 - 3 } Cat-II & $2(22.22)$ & $1(11.11)$ & \multirow{2}{*}{0.789} \\
\cline { 1 - 3 } Cat-III & $2(22.22)$ & $3(33.33)$ & \\
\hline Cat-IV & $3(33.33)$ & $1(11.11)$ & \\
\cline { 1 - 3 } Table 1. CEUS for Detection of Thyroid Malignancy \\
\hline
\end{tabular}

\section{DISCUSSION}

CEUS performed with the use of a microbubble contrast agent may be a potentially useful adjunct in assessing thyroid nodules, since it has a high specificity of $84.8 \%$ and high sensitivity of $76.9 \%$. Previous studies have demonstrated the feasibility of contrast-enhanced ultrasonography (CEUS) for the differentiation of benign and malignant thyroid nodules. ${ }^{(9)}$ Nemec et al reported that the complete CEUS data of 42 patients $(73.8 \%)$, benign and (26.2\%) malignant nodules revealed a significant difference in enhancement between benign and malignant nodules. Furthermore, CEUS demonstrated sensitivity of $76.9 \%$, specificity of $84.8 \%$ and accuracy of $82.6 \%$. Quantitative analysis of CEUS using a microbubble contrast agent allows the differentiation of benign and malignant thyroid nodules and may potentially serve in addition to gray-scale and Doppler ultrasound as an adjunctive tool in the assessment of patients with thyroid nodules.(2) Future studies should compare these findings to benign pathologies in order to establish CEUS as a standard diagnostic procedure in the preoperative evaluation of suspicious thyroid nodules.(3) Zhang reported that contrastenhanced US enhancement patterns were different in benign and malignant lesions. Ring enhancement was predictive of benign lesions, whereas heterogeneous enhancement was helpful for detecting malignant lesion.

\section{Strength and Limitation}

CEUS can be a definite value added tool and real-time costeffective and least adverse reaction is the expected modality in detecting solitary thyroid nodule. CEUS to replace FNAC in future. As our study was done on small sample volume, limitations can overcome by further large cohort study in future to prove CEUS modality of choice.

\section{CONCLUSION}

CEUS prove definitely adjunct to routine real-time ultrasonography in challenging and problem solving solitary thyroid nodule. Future prospects are yet to be defined for CEUS to include as value added tool along with USG on routine Ultrasonography screening.

\section{ACKNOWLEDGEMENT}

We thank Venkatesan Zigma, PhD, for the help with statistical analysis. We also thank the participants without whom this would not have been possible.

\section{REFERENCES}

[1] La Vecchia C, Malvezzi M, Bosetti C, et al. Thyroid cancer mortality and incidence: a global overview. Int J Cancer 2015;136(9):2187-95.

[2] Wang Y, Wang W. Increasing incidence of thyroid cancer in Shanghai, China, 1983-2007. Asia Pac J Public Health 2015;27(2):NP223-9.

[3] Morris LF, Ragavendra N, Yeh MW. Evidence-based assessment of the role of ultrasonography in the management of benign thyroid nodules. World J Surg 2008;32(8):1253-63.

[4] Tamsel S, Demirpolat G, Erdogan M, et al. Power doppler US patterns of vascularity and spectral doppler US parameters in predicting malignancy in thyroid nodules. Clin Radiol 2007;62(3):245-51.

[5] Kim JY, Lee CH, Kim SY, et al. Radiologic and pathologic findings of nonpalpable thyroid carcinomas detected by ultrasonography in a medical screening center. J Ultrasound Med 2008;27(2):215-23.

[6] Vigneri R, Malandrino P, Vigneri P. The changing epidemiology of thyroid cancer: why is incidence increasing? Curr Opin Oncol 2015;27(1):1-7.

[7] Lozano R, Naghavi M, Foreman K, et al. Global and regional mortality from 235 causes of death for 20 age groups in 1990 and 2010: a systematic analysis for the global burden of disease study 2010. Lancet 2012;380(9859):2095-128.

[8] Aschebrook-Kilfoy B, Ward MH, Sabra MM, et al. Thyroid cancer incidence patterns in the United States by histologic type, 1992-2006. Thyroid 2011;21(2):125-34.

[9] Chen W, Zheng R, Zeng H, et al. Annual report on status of cancer in China, 2011. Chin J Cancer Res 2015;27(1):2-12. 